Research Article

\title{
Levosimendan Protects against Doxorubicin-Induced Cardiotoxicity by Regulating the PTEN/Akt Pathway
}

\author{
Ling-Li Li, ${ }^{1,2}$ Li Wei, ${ }^{1,2,3}$ Ning Zhang, ${ }^{1,2}$ Wen-Ying Wei, ${ }^{1,2}$ Can Hu, ${ }^{1,2}$ Wei Deng $\mathbb{D}^{1,2,4}$ \\ and Qi-Zhu Tang ${ }^{1,2}$ \\ ${ }^{1}$ Department of Cardiology, Renmin Hospital of Wuhan University, Wuhan 430060, China \\ ${ }^{2}$ Hubei Key Laboratory of Metabolic and Chronic Diseases, Wuhan 430060, China \\ ${ }^{3}$ Department of Pediatrics, Renmin Hospital of Wuhan University, Wuhan 430060, China \\ ${ }^{4}$ Department of Cardiology, The Fifth Affiliated Hospital of Xinjiang Medical University, Ürümqi, China
}

Correspondence should be addressed to Wei Deng; vivideng1982@whu.edu.cn and Qi-Zhu Tang; qztang@whu.edu.cn

Received 7 March 2020; Revised 22 May 2020; Accepted 23 May 2020; Published 8 June 2020

Academic Editor: Oreste Gualillo

Copyright (c) 2020 Ling-Li Li et al. This is an open access article distributed under the Creative Commons Attribution License, which permits unrestricted use, distribution, and reproduction in any medium, provided the original work is properly cited.

Background and Aims. Myocyte apoptosis plays a critical role in the development of doxorubicin- (DOX-) induced cardiotoxicity. In addition to its cardiotonic effect, laboratory evidence indicates that levosimendan can inhibit apoptosis, but its role in DOX-induced cardiac injury remains unclear. Therefore, the present study is aimed at exploring whether levosimendan could attenuate DOX-induced cardiotoxicity. Methods. Levosimendan $(1 \mathrm{mg} / \mathrm{kg})$ was administered to mice through oral gavage once daily for 4 weeks, and the mice were also subjected to an intraperitoneal injection of DOX ( $5 \mathrm{mg} / \mathrm{kg})$ or saline, once a week for 4 weeks, to create a chronic model of DOX-induced cardiotoxicity. A morphological examination and biochemical analysis were used to evaluate the effects of levosimendan. H9C2 cells were used to verify the protective role of levosimendan in vitro. And an Akt inhibitor was utilized to verify the cardioprotection of levosimendan. Results. Levosimendan reduced the cardiac dysfunction and attenuated the myocardial apoptosis induced by DOX in vivo and in vitro. Levosimendan also inhibited the activation of phosphatase and tensin homolog (PTEN) and upregulated P-Akt expression both in vivo and in vitro. And inhibition of Akt abolished the cardioprotection of levosimendan in vitro. Conclusion. Levosimendan may protect against DOX-induced cardiotoxicity via modulation of the PTEN/Akt signaling pathway.

\section{Introduction}

In the past few decades, cancer mortality rates have declined, and the side effects caused by anticancer chemotherapeutic agents have become increasingly prominent. Doxorubicin (DOX) is a broad-spectrum anthracycline antibiotic used to treat solid and hematogenous malignancies [1-3]. The cumulative and dose-dependent toxicity induced by DOX is harmful to nontumor tissues, and in myocardial tissue, DOX causes irreversible damage, which can lead to dilated cardiomyopathy, greatly limiting its clinical application $[4,5]$. Many mechanisms underlying DOX-induced cardiotoxicity have been discovered, including mitochondrial iron accumulation and related redox reactions, the activation of immunological reactions, histamine release, and DNA damage, and emerging research indicates a crucial role for apoptosis [6-8].

Levosimendan, a calcium sensitizer, is known as a promising positive inotropic and vasodilatory agent used in the treatment of acute heart failure and other circumstances where an improvement in hemodynamics is required [9-12]. Levosimendan may exert its protective effects through the modulation of reactive oxygen species formation, adenosine triphosphate-sensitive potassium channel activity, membrane potential, or the release of endothelial nitric oxide synthase-dependent nitric oxide [13-15]. In addition, previous studies have shown that levosimendan protects against oxidative injury in animal models via interference with apoptotic signaling [16]. Numerous studies have shown that Akt plays pivotal roles in protecting cardiac 
myocytes from damage by inhibiting apoptosis [16-18]. Akt, also named protein kinase $\mathrm{B}$, is a signaling nexus governing cell growth, cell proliferation, and cell survival [18, 19]. Activation of Akt is regulated by upstream phosphatase and tensin homolog (PTEN), which reduces the phosphorylation of Akt and blocks downstream signaling events regulated by Akt [19-21]. Therefore, the aim of the current study was to investigate the protective effect and the mechanism of levosimendan in DOX-induced cardiotoxicity.

\section{Materials and Methods}

All animal experiments were performed in accordance with the Guide for the Care and Use of Laboratory Animals (National Institutes of Health publication number: 85-23, revised 1996) and approved by the Animal Care and Use Committee of Renmin Hospital of Wuhan University.

2.1. Reagents. DOX was purchased from Haizheng Pfizer Pharmaceutical Co., Ltd. Levosimendan was obtained from Orion Corporation, Espoo, Finland. The following primary antibodies were purchased from Cell Signaling Technology (Boston, MA, USA): Bcl-2-associated X protein (BAX; $1: 1000)$, c-caspase-3 (1:1000), PTEN (1:1000), P-Akt (1:1000), T-Akt (1:1000), and glyceraldehyde-3-phosphate dehydrogenase (GAPDH, $1: 1000)$, and B-cell lymphoma-2 (Bcl-2) $(1: 1000)$ was purchased from Abcam. Akt inhibitor (Akt i) was purchased from Sigma-Aldrich (St. Louis, MO, USA). A goat anti-rabbit secondary antibody was purchased from LI-COR Biosciences (Lincoln, USA). The BCA protein assay kit was obtained from Dōjindo Laboratories (Kumamoto, Japan).

2.2. Animals and Treatments. All animal care and experimental procedures were approved by the Animal Care and Use Committee of Renmin Hospital of Wuhan University, which is guided by the Guidelines for the Care and Use of Laboratory Animals published by the United States National Institutes of Health (NIH Publication, revised 2011). All the animal treatments and subsequent analysis were performed in a blind fashion for all groups. Male C57BL/6 mice ( 8 weeks old; body weight: $25.5 \pm 2$ g) were purchased from the Institute of Laboratory Animal Science, Chinese Academy of Medical Sciences (Beijing, China). The mice were allowed free access to food and water under a $12 \mathrm{~h}$ light-dark cycle with controlled temperature $\left(20-25^{\circ} \mathrm{C}\right)$ and humidity $(50 \pm 5 \%)$ in the Cardiovascular Research Institute of Wuhan University (Wuhan, China). A total of 40 male C57BL/6 mice were randomly divided into four groups: control group (CON, $n=10)$, control+levosimendan group $(\mathrm{CON}+\mathrm{L}$, $n=10$ ), doxorubicin group (DOX, $n=10$ ), and doxorubicin+levosimendan group (DOX $+\mathrm{L}, n=10)$. The mice were injected intraperitoneally with DOX $(5 \mathrm{mg} / \mathrm{kg}$, once a week; the total cumulative dose was $20 \mathrm{mg} / \mathrm{kg}$ ) or the same dose of saline for 4 weeks. Meanwhile, levosimendan $(1 \mathrm{mg} / \mathrm{kg})$ or the same volume of saline was administered through oral gavage once daily for 4 weeks. At the endpoint of the treatment, all the mice were anesthetized using $1.5 \%$ isoflurane and euthanized by cervical dislocation. The mouse hearts were dissected and weighed to assess heart weight/tibial length $(\mathrm{HW} / \mathrm{TL})$ and then snap-frozen in liquid nitrogen for further analysis.

2.3. Echocardiography and Hemodynamics. After the mice were anesthetized using isoflurane (1.5\%), echocardiography was performed using a MyLab 30CV ultrasound (Esaote SpA, Genoa, Italy) with a $10 \mathrm{MHz}$ linear array ultrasound transducer. The left ventricle (LV) end-systolic diameter (LVESD), LV end-diastolic dimension (LVEDD), LV ejection fraction (EF), and LV fractional shortening (FS) were measured along the short axis of the left ventricle at the level of the papillary muscles.

Hemodynamic variables were analyzed using a Millar catheter transducer (SPR-839; Millar Instruments, Houston, TX). The maximal rate of pressure development (dp/dtmax) and the minimal rate of pressure decay (dp/dtmin) were processed using PVAN data analysis software (Millar, Inc. Houston, TX, USA). All surgeries and analyses were performed in a blinded manner.

2.4. Morphological Analysis. The removed hearts were fixed in $10 \%$ formalin overnight. The hearts were transversely sectioned into $5 \mu \mathrm{m}$ slices. Then, the slices were stained using hematoxylin and eosin (HE). The cross-sectional area (CSA) of the cardiomyocytes was examined based on HE-stained sections using a quantitative digital image analysis system (Image-Pro Plus 6.0). More than 100 myocytes in the LV were outlined in each group.

2.5. Western Blotting and Quantitative Real-Time PCR. Total protein was extracted from the frozen heart tissues or H9C2 cells using RIPA agent (Invitrogen, Carlsbad, CA, USA). Then, the Pierce BCA Protein Assay Kit (23227, Thermo Scientific, MIT, USA) was used to measure the concentration of total protein. The proteins were separated through $10 \%$ dodecyl sulfate, sodium salt-polyacrylamide gel electrophoresis (SDS-PAGE) and then transferred to polyvinylidene fluoride (PVDF) membranes (cat. number IPFL00010; EMD Millipore, Billerica, MA, USA), and primary antibodies were incubated with the blot at $4^{\circ} \mathrm{C}$ overnight. After reaction with secondary antibodies at $37^{\circ} \mathrm{C}$ for $1 \mathrm{~h}$, the blots were scanned and analyzed using a two-color infrared imaging system (Odyssey, LICOR Biosciences, NE, USA). Total RNA was isolated using TRIzol and reverse-transcribed to cDNA. A LightCycler 480 SYBR Green Master Mix (cat. number 04896866001; Roche) was used to quantify amplification. The mRNA data were normalized to GAPDH.

2.6. Cell Culture. H9C2 cells were obtained from the Cell Bank of the Chinese Academy of Science (Shanghai, China) and were cultured in Dulbecco's modified Eagle's medium (DMEM, GIBCO, C11995), containing 10\% fetal bovine serum (GIBCO, 15140). H9C2 cells were seeded in six-well culture plates or on glass slides and incubated in $5 \% \mathrm{CO}_{2}$ and $95 \%$ air at $37^{\circ} \mathrm{C}$. The medium was exchanged for serum-free DMEM $48 \mathrm{~h}$ later, to starve the cells for $12 \mathrm{~h}$. Subsequently, the cells were pretreated using levosimendan $(10 \mu \mathrm{mol} / \mathrm{mL})$ or phosphate buffer saline (PBS) for $2 \mathrm{~h}$, and then, $\operatorname{DOX}(1 \mu \mathrm{mol} / \mathrm{L})$ was added into the medium in the 

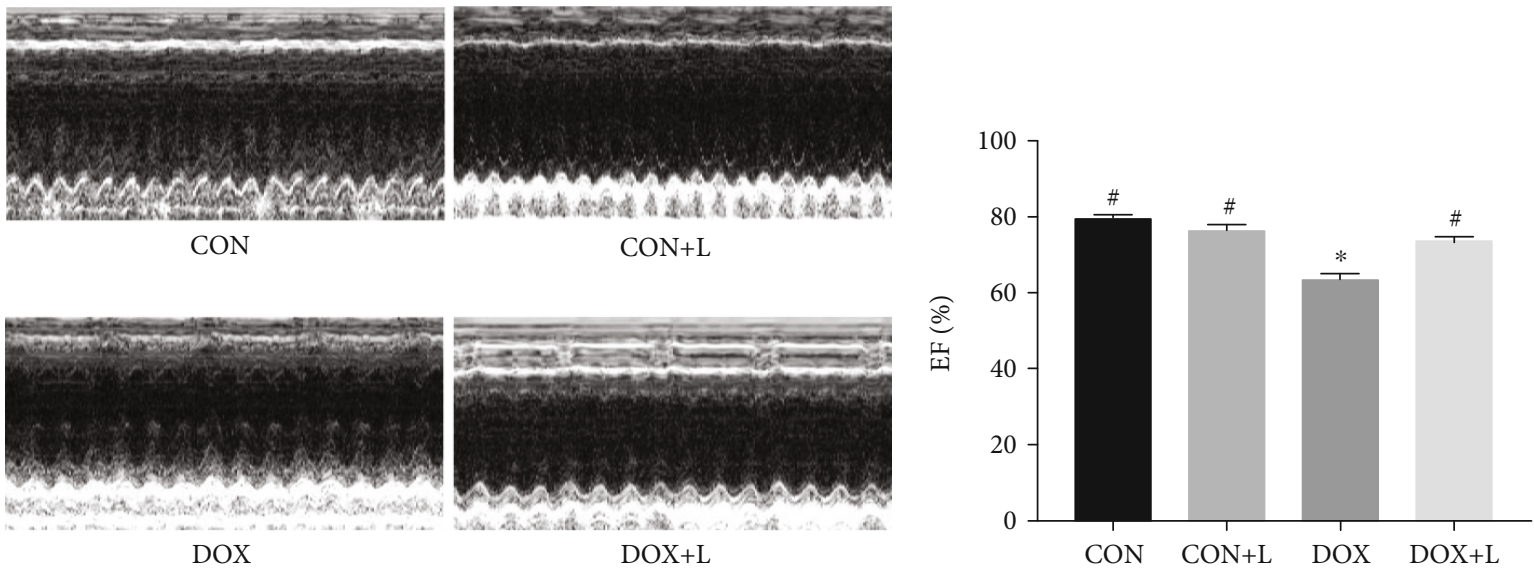

(a)

(b)

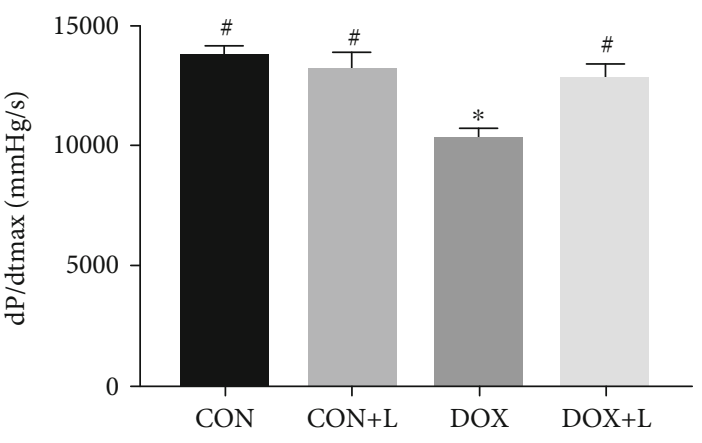

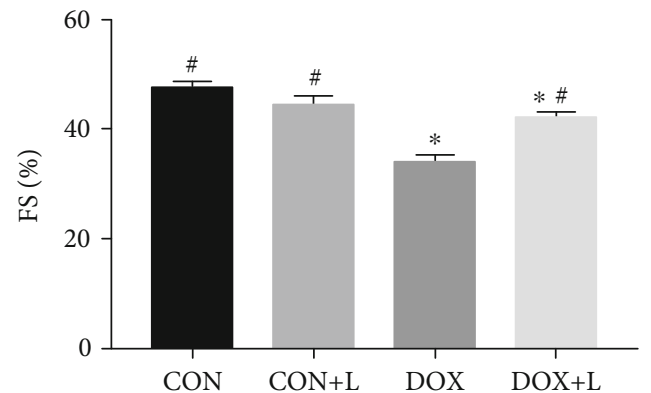

(c)

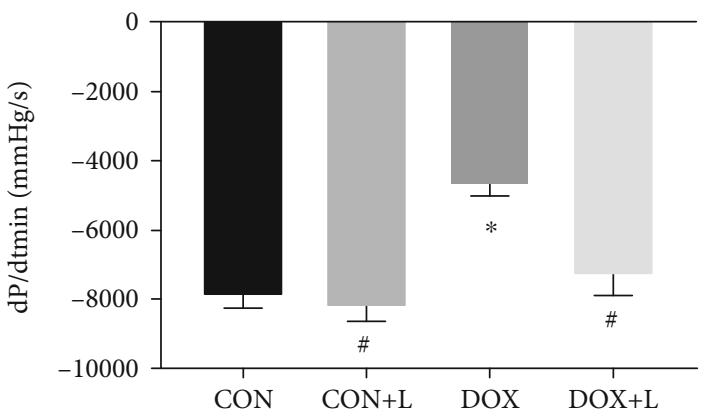

(d)

FIGURE 1: Levosimendan improved cardiac function in mice during DOX treatment. (a) Representative echocardiographic images of each group, including the CON, CON+L, DOX, and DOX+L groups (LVDs: left ventricular internal dimension systole; LVDd: left ventricular internal dimension diastole; IVS: interventricular septal thickness; PW: posterior wall). (b) Left ventricular ejection fraction (EF) of mice with or without treatment with levosimendan 4 weeks after injection of DOX or saline. (c) Fractional shortening (FS) in each group of mice. (d) Hemodynamic analysis of mice including dp/dt max and dp/dt min. ${ }^{*} P<0.05$, vs. the CON group; ${ }^{*} P<0.05$, vs. the DOX group.

presence or absence of levosimendan for $24 \mathrm{~h}$. Proteins or mRNA were then harvested. Meanwhile, H9C2 cells were pretreated with AKT i $(1 \mu \mathrm{mol} / \mathrm{L})$ for $30 \mathrm{~min}$, as we previously described [22].

2.7. Terminal Deoxynucleotidyl Transferase-Mediated Nick End Labeling (TUNEL) Staining. Briefly, terminal deoxynucleotidyl transferase-mediated nick end labeling (TUNEL) staining was performed using a commercial kit (Millipore, Billerica, MA, USA) in accordance with the manufacturer's instructions. The nuclei of the cardiac myocytes were stained using $4^{\prime}$,6-diamidino-2-phenylindole (DAPI). Subsequently, slices of paraffin-embedded tissue or cells were observed and images were captured using an Olympus DX51 fluorescence microscope (Olympus, Japan).

2.8. Statistical Analysis. All data are presented as the mean \pm SD. A one-way analysis of variance followed by Tukey's post hoc test was used to analyze differences among groups. $P<0.05$ was considered to indicate statistical significance.

\section{Results}

3.1. Levosimendan Improved Cardiac Function in Mice Undergoing DOX Treatment. After 4 weeks, all 40 mice 


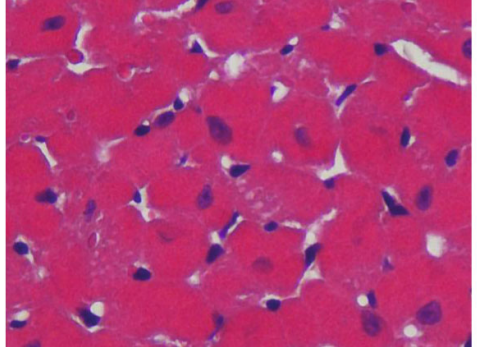

$\mathrm{CON}$

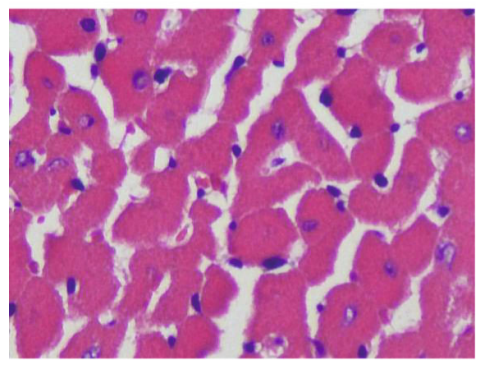

DOX

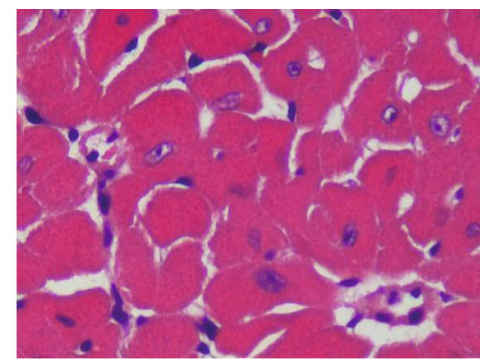

$\mathrm{CON}+\mathrm{L}$

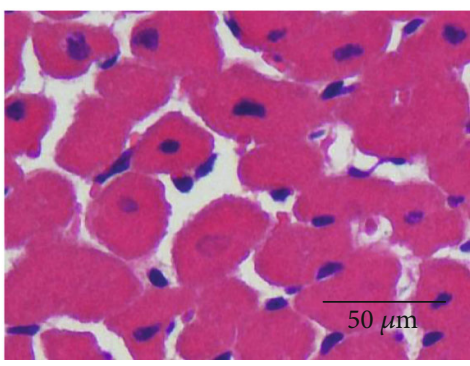

$\mathrm{DOX}+\mathrm{L}$

(a)
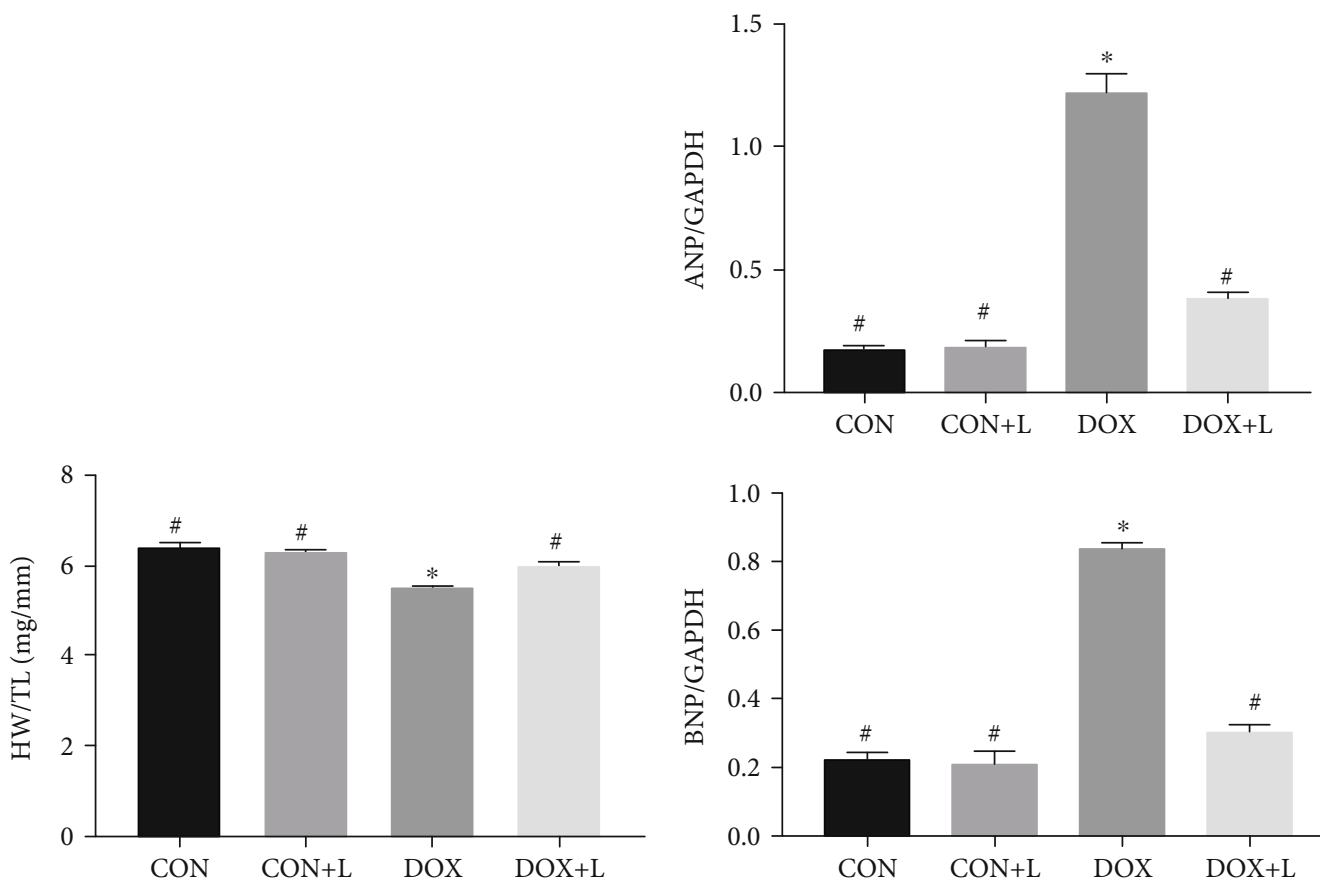

(c)

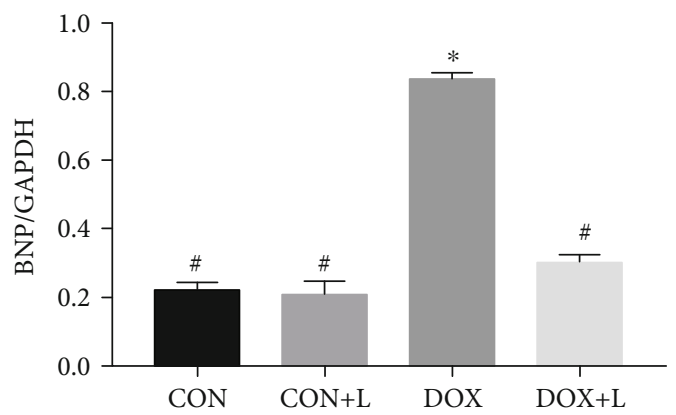

(d)

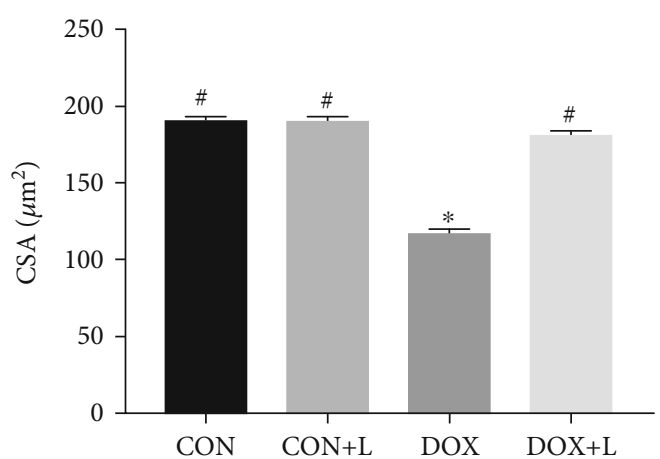

(b)

FIGURE 2: Levosimendan ameliorated the myocardial damage induced by DOX. (a) HE staining and representative images in mice. Scale bar: $50 \mu \mathrm{m}$. (b) Statistical analysis of cross-sectional area (CSA, $n=100+$ cells per experimental group). (c) The ratio of heart weight and tibia length (HW/TL) in each group. (d) Real-time PCR for the mRNA expression of cardiac injury-associated genes from the myocardium in each group, including ANP and BNP (normalized to GAPDH). ${ }^{*} P<0.05$, vs. the CON group; ${ }^{*} P<0.05$, vs. the DOX group.

survived. To determine the effect of levosimendan on cardiac dysfunction, the wall thickness, chamber diameter, and LV function were measured. As shown in Figure 1(a), the wall thickness of the DOX group was thinner and the LVEDD was larger than that of the CON and CON $+\mathrm{L}$ groups, and these changes were reversed slightly in the $\mathrm{DOX}+\mathrm{L}$ group, although these differences were not significant (Figure 1(a)). Echocardiography and hemodynamic measurements demonstrated that DOX reduced cardiac systolic heart function, as demonstrated by decreased LVEF, LVFS, dp/dtmax, and dp/dtmin in DOX-treated mice. However, compared with the DOX group, the DOX $+\mathrm{L}$ group exhibited attenuated cardiac dysfunction, which was reflected by an improvement in LVEF, LVFS, dp/dtmax, and dp/dtmin (Figures 1(b)-1(d)). However, there was no difference in cardiac function between the CON and the CON+L groups. 

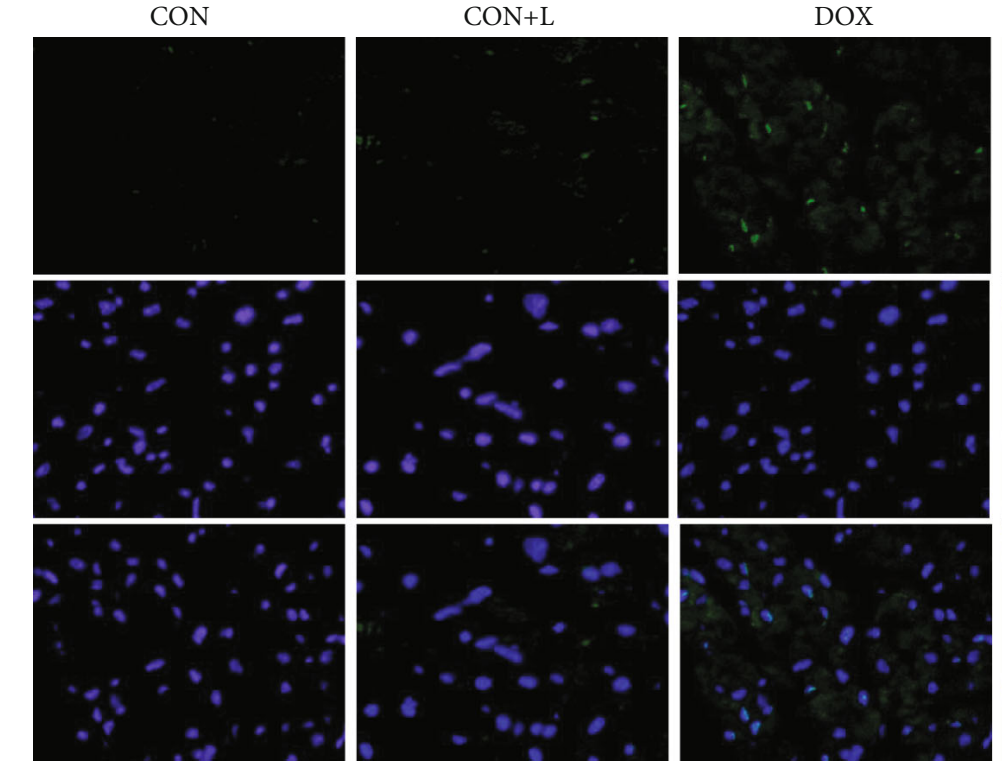

(a)

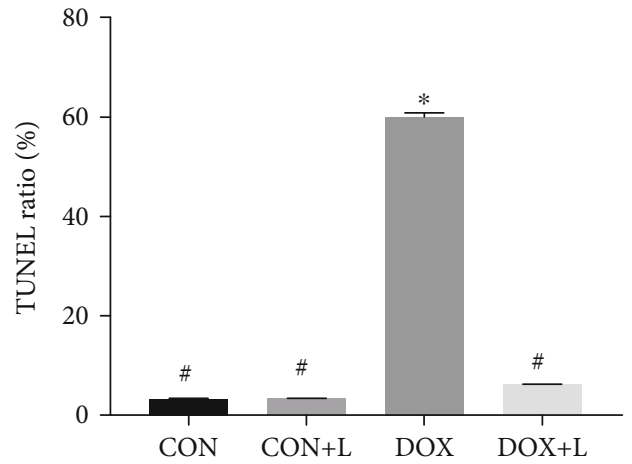

(b)
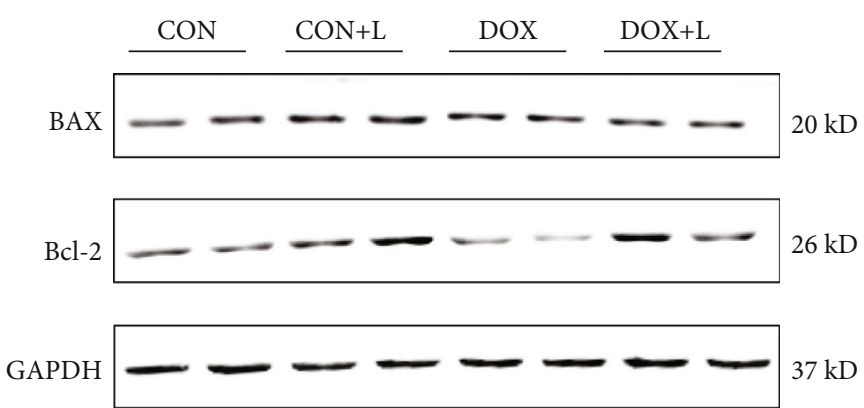

(c)

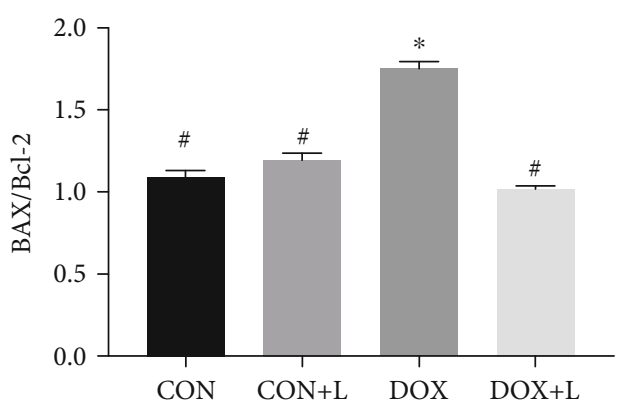

(d)

FIgURE 3: Levosimendan exhibited an antiapoptosis effect in DOX-treated mice. (a, b) Terminal deoxynucleotidyl transferase-mediated nick end labeling (TUNEL) assay for apoptosis in heart tissues and quantitation of the TUNEL ratio in the indicated group. Scale bar: $50 \mu \mathrm{m}$. (c, d) Representative western blots of BAX, Bcl-2, and c-caspase-3, and quantitative analysis for BAX/Bcl-2 and c-caspase-3/GAPDH ratio. ${ }^{*} P<$ 0.05 , vs. the CON group; ${ }^{*} P<0.05$, vs. the DOX group.

3.2. Levosimendan Ameliorated the Myocardial Injury Induced by DOX. To observe the effect of levosimendan on the myocardial injury caused by DOX treatment, HE staining and real-time PCR were performed. The results of HE staining indicated that the reduced CSA of myocardial cells following DOX injection was restrained by levosimendan (Figures 2(a) and $2(\mathrm{~b})$ ). In addition, levosimendan could attenuate the body weight loss induced by DOX treatment. DOX injection resulted in a decreased HW/TL, yet levosimendan could improve HW/TL (Figure 2(c)). Moreover, as determined through PCR, indexes of cardiac injury, including the mRNA expression of atrial natriuretic peptide (ANP) and brain natriuretic peptide (BNP), were increased in the DOX group and were mitigated in the DOX $+\mathrm{L}$ group (Figure $2(\mathrm{~d})$ ). 

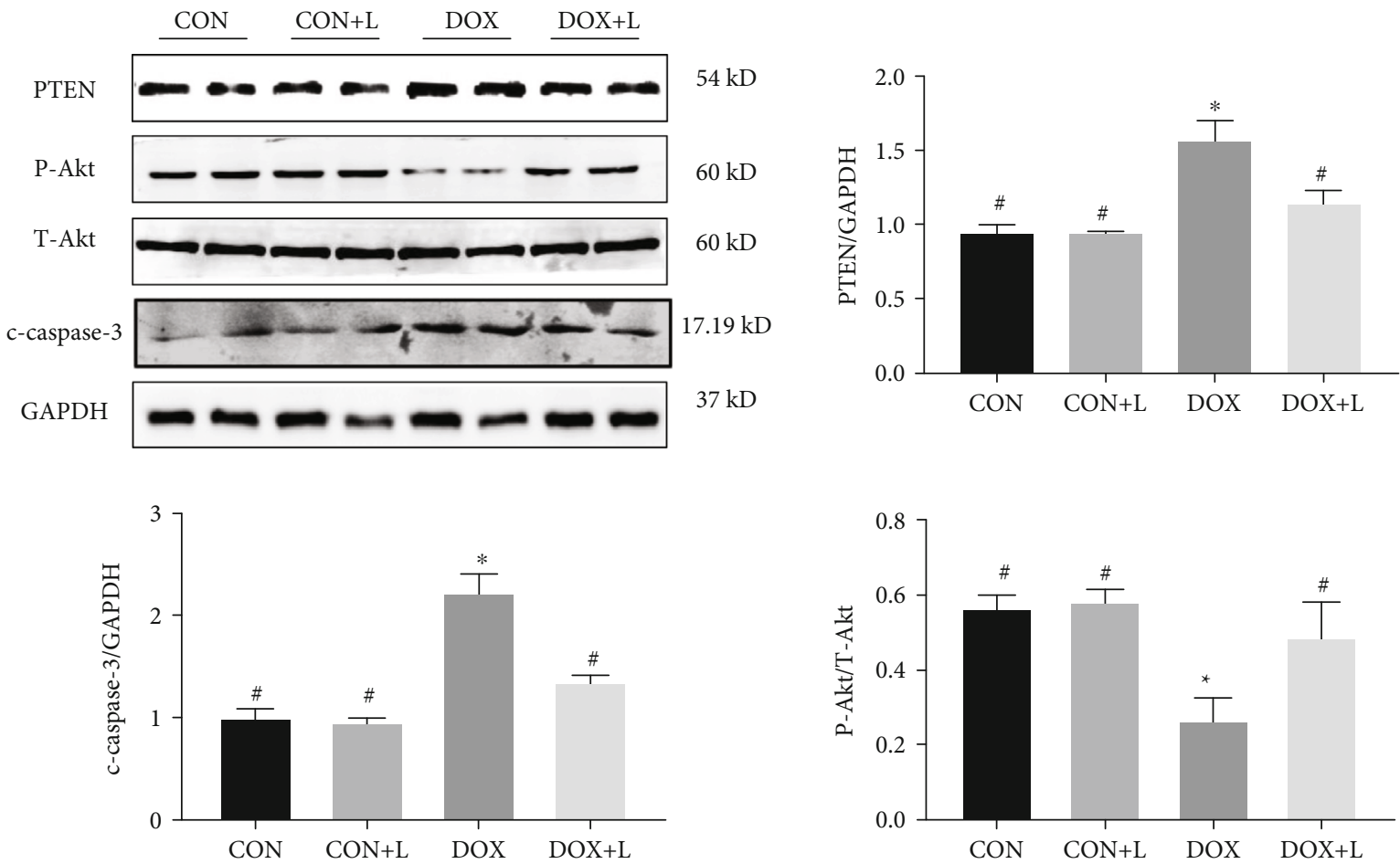

FIGURE 4: Levosimendan inhibited myocardial PTEN/Akt signaling in vivo. Western blotting and quantitative analysis of the PTEN/Akt pathway in cardiac tissue, including PTEN, P-Akt, and T-Akt protein level analysis. The ratio of PTEN/GAPDH and P-Akt/T-Akt was shown. ${ }^{*} P<0.05$, vs. the CON group; ${ }^{\#} P<0.05$, vs. the DOX group.

3.3. Levosimendan Exhibited an Antiapoptotic Effect in DOX-Treated Mice. To investigate the effects of levosimendan on myocardial apoptosis after DOX stimulation, TUNEL staining was performed. As shown in Figures 3(a) and 3(b), DOX injection resulted in a noteworthy increase in myocardial apoptosis, which was indicated by the increased number of TUNEL-positive cells, and levosimendan significantly restrained the apoptosis caused by DOX. Few TUNELpositive cells were detected in the CON group and the $\mathrm{CON}+\mathrm{L}$ group. We then measured the expression levels of apoptosis markers including $\mathrm{BAX}, \mathrm{Bcl}-2$, and c-caspase-3. We found that the injection of DOX resulted in increased proapoptotic BAX and c-caspase- 3 and reduced antiapoptotic Bcl-2 expression compared with no DOX injection. Levosimendan treatment could reverse these alterations (Figures 3(c) and 3(d)). The expression of BAX, Bcl-2, and c-caspase- 3 in the myocardium was not significantly altered in the $\mathrm{CON}$ and $\mathrm{CON}+\mathrm{L}$ groups.

\subsection{Levosimendan Inhibited Myocardial PTEN/Akt Signaling} In Vivo. To examine the underlying mechanism of levosimendan, the PTEN/Akt signaling pathway was examined through western blotting. The results showed that DOX injection remarkably upregulated the expression of PTEN as well as significantly downregulating P-Akt expression. However, levosimendan appeared to reduce the level of PTEN and ameliorated the expression of P-Akt (Figure 4). These results suggest that levosimendan might protect against DOX-induced cardiotoxicity via inhibition of the PTEN/Akt pathway.
3.5. Levosimendan Suppressed DOX-Induced Cardiotoxicity via the PTEN/Akt Pathway In Vitro. To verify the protective effect of levosimendan in DOX-induced cardiotoxicity and its molecular mechanism in vitro, cell experiments were performed. As shown in Figure 5(a), many TUNEL-positive cells were observed in $\mathrm{H} 9 \mathrm{C} 2$ cells in the DOX group; in contrast, in the DOX $+\mathrm{L}$ group, TUNEL-positive cells were much less abundant (Figure 5(a)). In addition, western blot analyses revealed that the expression of proapoptotic BAX and c-caspase-3 was increased while that of antiapoptotic Bcl-2 was decreased in the DOX group compared with the CON group. Levosimendan could reverse these changes (Figure 5(c)). Levosimendan could also ameliorate DOXinduced cardiomyocyte injury, which was reflected by a decrease in ANP and BNP compared with the DOX group (Figure 5(b)). Furthermore, the PTEN/Akt signaling pathway, a key mechanism regulating the progression of cell growth and apoptosis, was inhibited by levosimendan in vitro.

3.6. Inhibition of Akt Abolished the Protective Effect of Levosimendan. To investigate the PTEN/Akt signaling pathway in levosimendan-mediated protective effect during DOX, we pretreated $\mathrm{H} 9 \mathrm{C} 2$ cells with the Akt inhibitor. As is shown in Figure 6(a), Akt inhibitor administration significantly decreased the expression of Akt in $\mathrm{H} 9 \mathrm{C} 2$ cells. Then, we detected the cardiomyocyte injury and apoptosis. Expectedly, levosimendan evidently blocked H9C2 injury attributed by DOX; however, coadministration with Akt $i$ eliminated the optimistic effect of levosimendan 

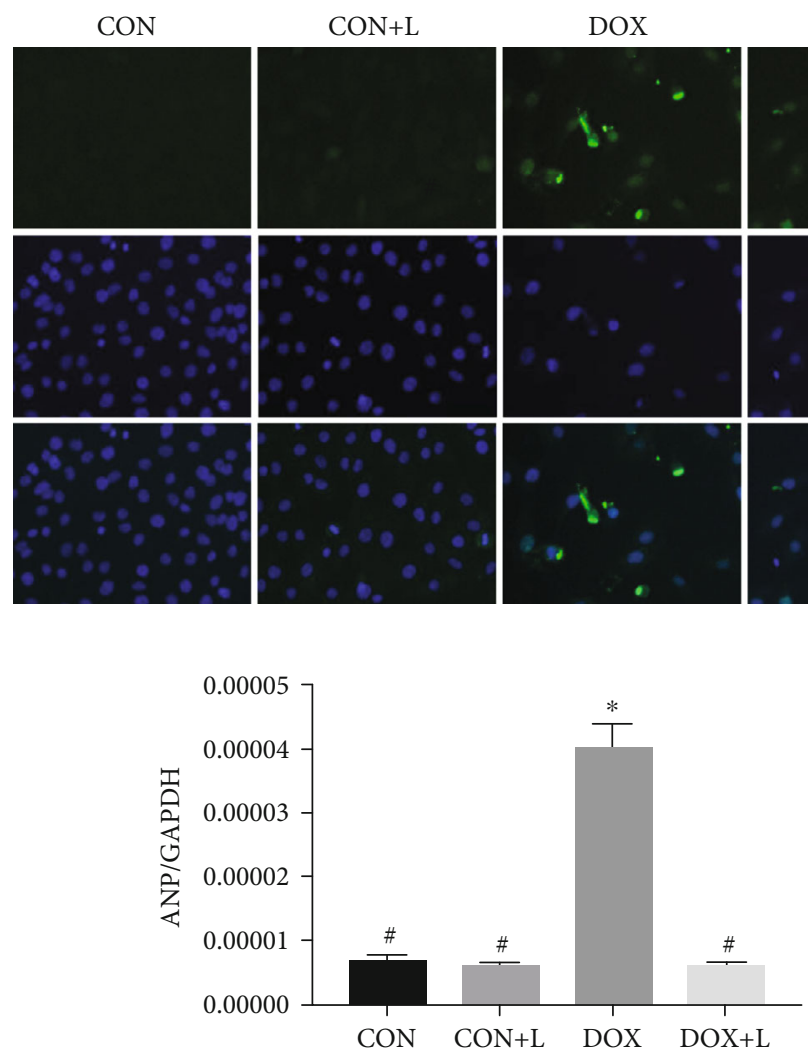

$\mathrm{DOX}+\mathrm{L}$
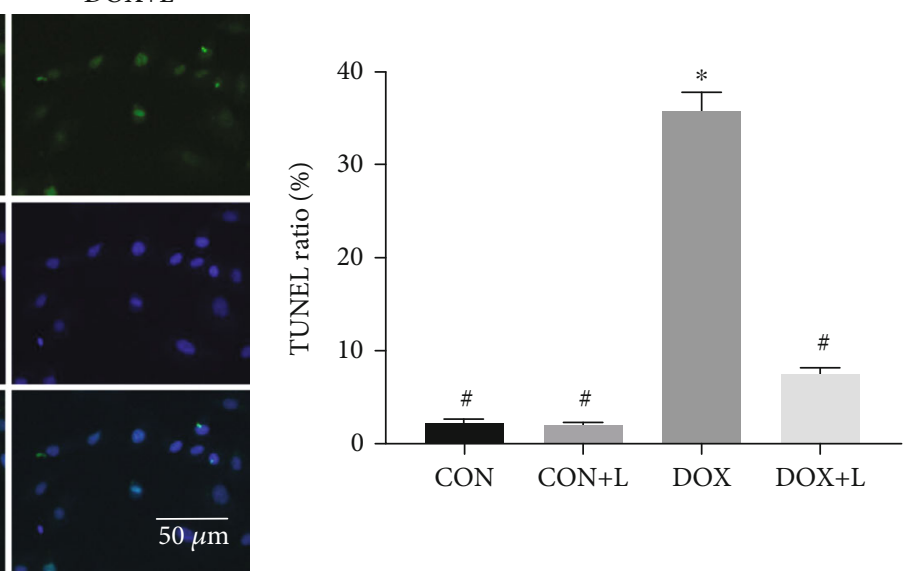

(a)

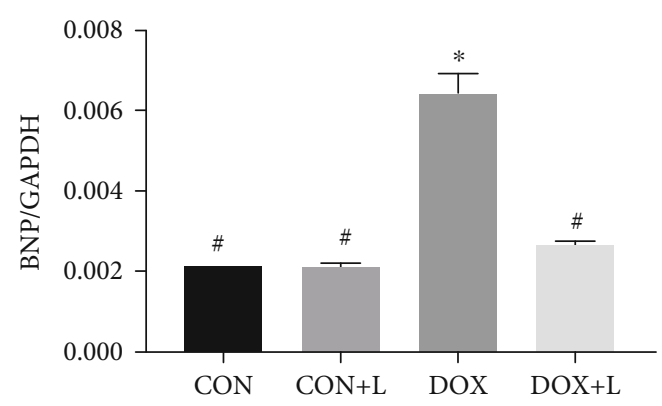

(b)
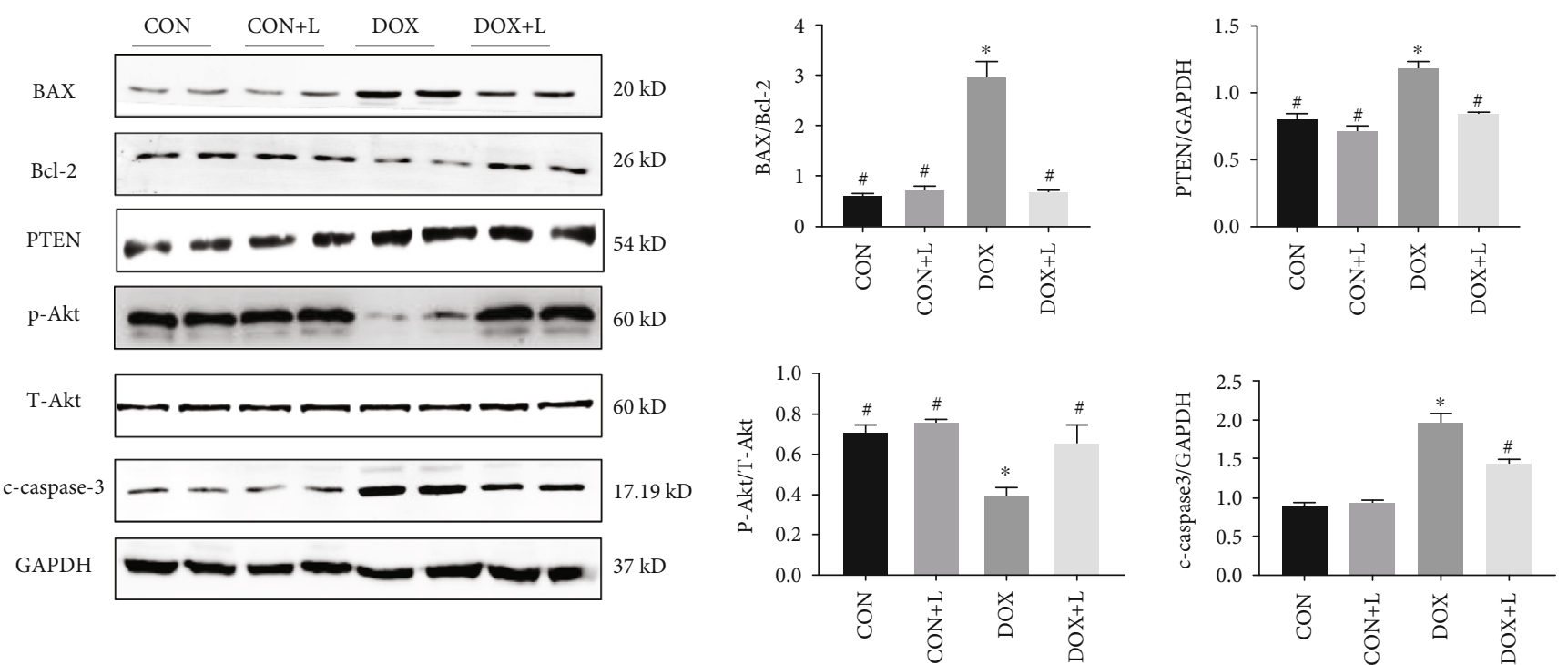

(c)

FIgURE 5: Levosimendan suppressed DOX-induced cardiotoxicity via the PTEN/Akt pathway in vitro. (a) TUNEL staining for apoptosis in H9C2 cells and quantitative analysis of the TUNEL ratio in each group. Scale bar: $50 \mu \mathrm{m}$. (b) Real-time PCR for the mRNA expression of cardiac injury-associated genes in H9C2 cells in each group, including ANP and BNP (normalized to GAPDH). (c) Western blotting and quantitative analysis of apoptosis-related proteins and the PTEN/Akt signaling pathway in H9C2 cells, including BAX, Bcl-2, c-caspase-3, PTEN, P-Akt, and T-Akt. GAPDH was used as a loading control. ${ }^{*} P<0.05$, vs. the CON group; ${ }^{\#} P<0.05$, vs. the DOX group.

(Figure 6(b)). Additionally, the analyses of TUNEL indicated that levosimendan alleviated the apoptosis induced by DOX, nevertheless, Akt i removed the function of levo- simendan (Figure 6(c)). These data suggested that Akt inhibition abolished the cardioprotection of levosimendan during DOX. 

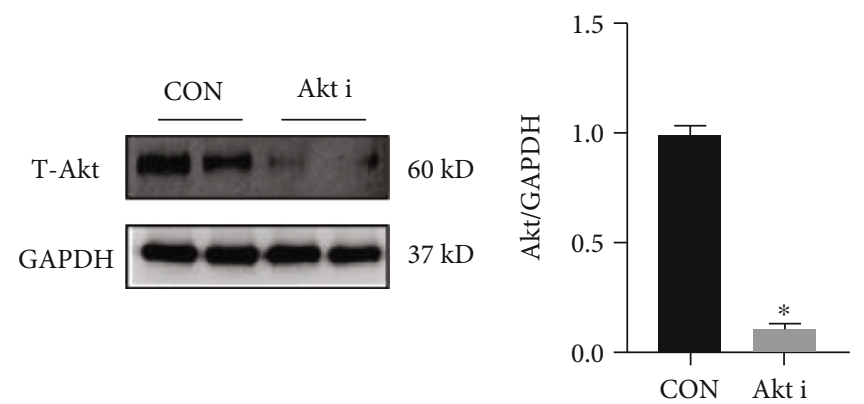

(a)
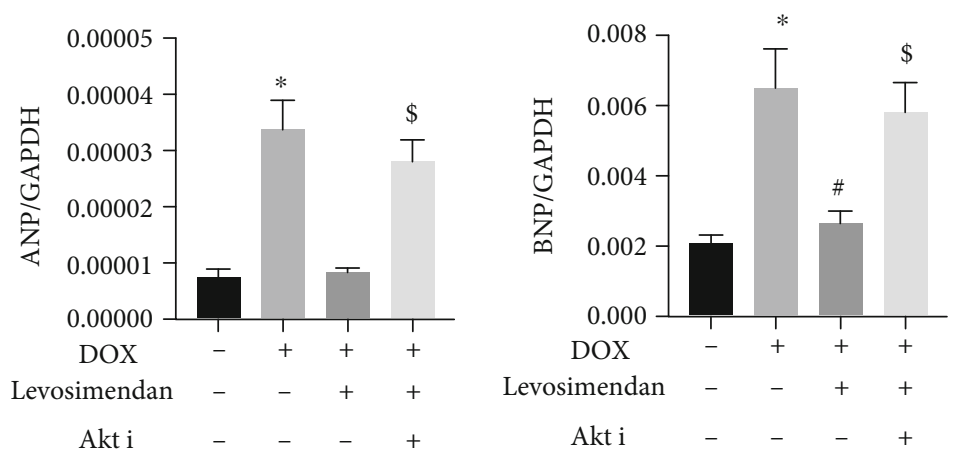

(b)
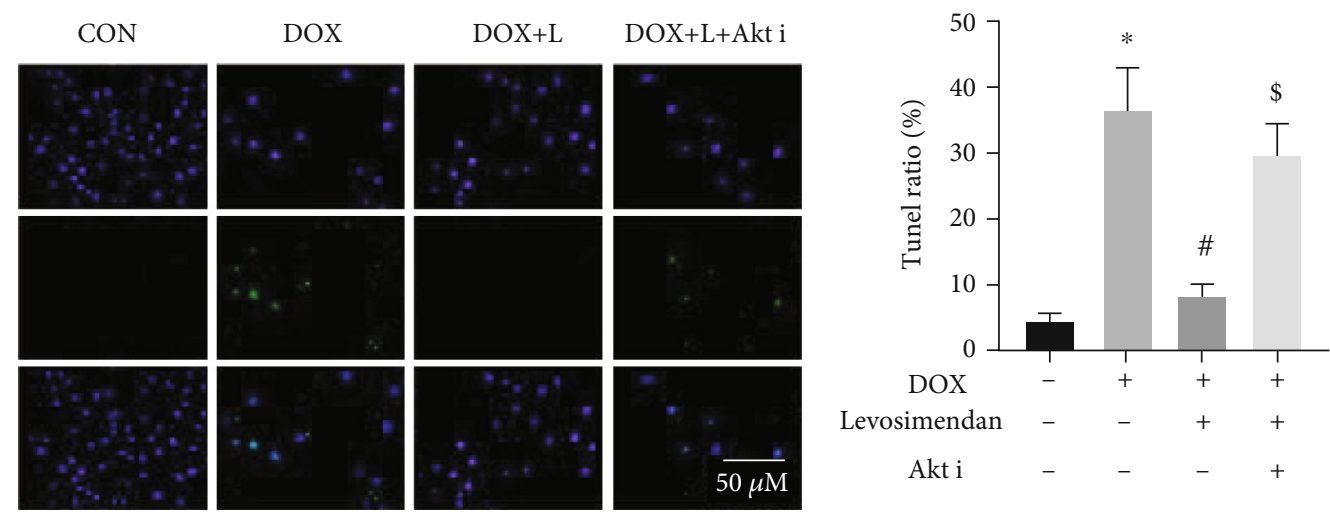

(c)

FIGURE 6: Inhibition of Akt abolished the protective effect of levosimendan in vitro. (a) Western blotting and quantitative analysis of Akt in H9C2 cells. (b) Real-time PCR for the mRNA expression of cardiac injury-associated genes in H9C2 cells in each group, including ANP and BNP (normalized to GAPDH). (c) TUNEL staining for apoptosis in H9C2 cells and quantitative analysis of the TUNEL ratio in each group. Scale bar: $50 \mu \mathrm{m} .{ }^{*} P<0.05$, vs. the CON group; ${ }^{*} P<0.05$, vs. the DOX group; ${ }^{\$} P<0.05$, vs. the DOX $+\mathrm{L}$ group.

\section{Discussion}

In the current study, we demonstrated that levosimendan could attenuate DOX-induced cardiotoxicity by significantly protecting cardiac function and reducing myocardial injury and apoptosis in vivo and in vitro. Furthermore, we demonstrated that these protective effects were mediated by the inhibition of PTEN and the subsequent activation of Akt, both in vivo and in vitro. Herein, the current study provided a novel approach for the treatment of DOXinduced cardiotoxicity.

Apoptosis has been shown to be one of the key processes in DOX-induced cardiac injury $[4,8,23,24]$. We observed few apoptotic cells in the CON and CON+L groups, but more apoptotic cells in the DOX group. Apoptosis, characterized by the orderly death of cells, was controlled by correlative genes, and apoptosis can be induced by many stimuli such as hypoxia, inflammation, and related drugs [23, 25]. Apoptosis is an active process that enables adaptation to external factors rather than a passive process, and excessive apoptosis is unfavorable. There are two relatively clear apoptotic pathways: the external pathway involves cleavage of the initiator procaspase- 8 into the active caspase- 8 , thereby causing a series of apoptosis cascades once the Fas ligand binds to the Fas receptor on the cell surface $[22,26,27]$; and the endogenous pathway is strictly regulated by proteins from the Bcl-2 family and targets mitochondria, maintaining the competition between antiapoptotic Bcl-2-like survival factors and 
proapoptotic BAX-like death factors $[22,27,28]$. BAX and $\mathrm{Bcl}-2$ belong to the family of $\mathrm{Bcl}-2$ proteins. If $\mathrm{BAX}$ expression is increased, a homodimer of BAX/BAX is produced which promotes apoptosis; otherwise, a heterodimer or homodimer of $\mathrm{Bcl}-2 / \mathrm{Bcl}-2$ is generated to inhabit apoptosis. The ratio of those two factors determines whether apoptosis occurs, as well as the severity of apoptosis. Furthermore, as the cytokines involved in the regulation of apoptosis, caspases are core participators in the activation of apoptotic cascades [16, 27, 28]. The concentration of active caspase- 3 is closely associated with the rate of apoptosis. The current study showed that the expression of BAX and active caspase- 3 proteins in the DOX group was upregulated dramatically both in vivo and in vitro and that the $\mathrm{Bcl}-2 / \mathrm{BAX}$ ratio decreased significantly. In addition, the expression of $\mathrm{BAX}$ and $\mathrm{c}$-caspase -3 in the $\mathrm{CON}+\mathrm{L}$ group was decreased.

DOX activates PTEN, thereby modulating the activity downstream of the PI3K/Akt pathway [27]. PTEN mutation is the most common mutation in tumor suppressor genes which may negatively regulate cell function [29-31]. It has been universally acknowledged that the most important role of PTEN is to regulate growth and development, including cell growth, adhesion, migration, invasion, and apoptosis, and it also serves as the key regulator of pathogenesis in a variety of cardiovascular diseases (CVD), probably via the PI3K/Akt signaling pathway [32-36]. Multiple physical and chemical stimuli can activate PI3K and thereby phosphorylate-Akt ( $\mathrm{p}$-Akt), which subsequently functions as a key factor in downstream effectors [37, 38]. Activated Akt may exert its antiapoptotic effect through the regulation of caspase-3. Caspase-3 is a vital effector of cell apoptosis, existing as a precursor under normal conditions, and it is activated during apoptosis. Procaspase- 3 can be activated through cleavage, and activated caspase- 3 is the most important terminal cleavage enzyme in apoptosis [39-41]. Early investigations suggested that the activation of Akt could effectively inhibit cardiomyocyte apoptosis and reduce the extent of myocardial injury. In the current study, we have shown that the PTEN/Akt pathway was activated in DOXinduced apoptosis.

Regarding levosimendan, previously reported experimental results have indicated that it could increase the sensitivity between $\mathrm{Ca}^{2+}$ and myofilaments by combining with myocardial troponin $\mathrm{C}$ to strengthen myocardial contraction without increasing the intracellular $\mathrm{Ca}^{2+}$ concentration and oxygen consumption in the myocardium $[13,16,27]$. Simultaneously, its vasodilatory and anti-ischemic effects might be mediated by the opening of adenosine triphosphatesensitive potassium channels in vascular smooth muscle cells. Moreover, levosimendan may protect various organs, including the heart, kidney, lung, and liver, from apoptotic cell death, probably through the modulation of membrane potential, reactive oxygen species formation, and adenosine triphosphate-sensitive potassium channel activity, thereby regulating mitochondrial function [42-44]. Recently, it has been reported that levosimendan can significantly inhibit interleukin- $\beta$-induced apoptosis in adult rat cardiac fibroblasts. This cytoprotective effect of levosimendan was sug- gested to be caused by the activation of Akt and the inhibition of inducible nitric oxide synthase (iNOS) expression and subsequent NO production [16]. In addition, a recent experiment showed that levosimendan pretreatment was associated with attenuated myocardial apoptosis and therefore partially reverses coronary microembolization-induced myocardial dysfunction in swine. The study also revealed that the potential mechanisms underlying the protective effects of levosimendan in coronary microembolization-induced cardiac dysfunction might involve the regulation of Akt [27].

In conclusion, the data in the current study demonstrate that levosimendan relieves DOX-induced myocardial injury and improves cardiac function. These protective effects involve regulation of the PTEN/Akt signaling pathway, which leads to reduced apoptosis. And levosimendan therapy targeting the PTEN/Akt pathway may be a promising therapeutic approach to treat chemotherapeutic agent-induced cardiotoxicity.

\section{Data Availability}

Requests by researchers to access the data, analytic methods, and study materials for the purposes of reproducing the results or replicating procedures can be made to the corresponding author who manages the information or by contacting the corresponding authors (Deng, Wei (email: vivideng1982@whu.edu.cn) and Tang, Qi-Zhu (email: qztang@whu.edu.cn), address: Department of Cardiology, Renmin Hospital of Wuhan University, Jiefang Road 238, Wuhan 430060, China, Tel.: +86 27 88073385; fax: +86 27 88042292).

\section{Conflicts of Interest}

The authors declare that they have no conflicts of interest.

\section{Authors' Contributions}

Ling-Li Li and Li Wei contributed equally to this work.

\section{Acknowledgments}

This work was supported by grants from the National Key R\&D Program of China (2018YFC1311300), the Key Project of the National Nature Science Foundation of China (No. 81530012), the Fundamental Research Funds for the Central Universities (No. 2042019kf0094), and the National Nature Science Foundation of China (Nos. 81860080 and 81470516).

\section{References}

[1] H. He, Y. Luo, Y. Qiao et al., "Curcumin attenuates doxorubicin-induced cardiotoxicity via suppressing oxidative stress and preventing mitochondrial dysfunction mediated by 14-3-3 $\gamma$," Food \& Function, vol. 9, no. 8, pp. 4404-4418, 2018.

[2] G. Pakravan, A. M. Foroughmand, M. Peymani et al., "Downregulation of miR-130a, antagonized doxorubicin-induced cardiotoxicity via increasing the _PPAR $\gamma$ expression in mESCs-derived cardiac cells," Cell Death \& Disease, vol. 9, no. 7 , p. 758, 2018. 
[3] J. M. dos Santos, T. M. Alfredo, K. Á. Antunes et al., "Guazuma ulmifolia Lam. decreases oxidative stress in blood cells and prevents doxorubicin-induced cardiotoxicity," Oxidative Medicine and Cellular Longevity, vol. 2018, 16 pages, 2018.

[4] Q. Dong, L. Chen, Q. Lu et al., "Quercetin attenuates doxorubicin cardiotoxicity by modulating Bmi-1 expression," British Journal of Pharmacology, vol. 171, no. 19, pp. 4440-4454, 2014.

[5] F. Cochera, D. Dinca, D. A. Bordejevic et al., "Nebivolol effect on doxorubicin-induced cardiotoxicity in breast cancer," Cancer Management and Research, vol. 10, pp. 2071-2081, 2018.

[6] M. Kuzu, F. M. Kandemir, S. Yildirim, S. Kucukler, C. Caglayan, and E. Turk, "Morin attenuates doxorubicininduced heart and brain damage by reducing oxidative stress, inflammation and apoptosis," Biomedicine \& Pharmacotherapy, vol. 106, pp. 443-453, 2018.

[7] X. Zhao, Y. Yang, F. Yao et al., "Unfolded protein response promotes doxorubicin-induced nonsmall cell lung cancer cells apoptosis via the mTOR pathway inhibition," Cancer Biotherapy and Radiopharmaceuticals, vol. 31, no. 10, pp. 347-351, 2016.

[8] Y.-J. Jeon, J.-I. Shin, S. Lee et al., "Angelica gigas Nakai has synergetic effects on doxorubicin-induced apoptosis," BioMed Research International, vol. 2018, 11 pages, 2018.

[9] B. Gong, Z. Li, and P. C. Yat Wong, "Levosimendan Treatment for Heart Failure: A Systematic Review and Meta- Analysis," Journal of Cardiothoracic and Vascular Anesthesia, vol. 29, no. 6, pp. 1415-1425, 2015.

[10] H. A. Kiraz, F. Poyraz, G. Kip et al., "The effect of levosimendan on myocardial ischemia-reperfusion injury in streptozotocin-induced diabetic rats," Libyan Journal of Medicine, vol. 10, no. 1, p. 29269, 2015.

[11] S. Yamashita, T. Suzuki, K. Iguchi et al., "Cardioprotective and functional effects of levosimendan and milrinone in mice with cecal ligation and puncture-induced sepsis," Naunyn-Schmiedeberg's Archives of Pharmacology, vol. 391, no. 9, pp. 10211032, 2018.

[12] V. Hillestad, F. Kramer, S. Golz, A. Knorr, K. B. Andersson, and G. Christensen, "Long-term levosimendan treatment improves systolic function and myocardial relaxation in mice with cardiomyocyte-specific disruption of the Serca2 gene," Journal of Applied Physiology, vol. 115, no. 10, pp. 15721580, 2013.

[13] Ş. C. Sezen, A. Küçük, A. Özer et al., "Assessment of the effects of levosimendan and thymoquinone on lung injury after myocardial ischemia reperfusion in rats," Drug Design, Development and Therapy, vol. 12, pp. 1347-1352, 2018.

[14] F. Q. Hinojosa, M. Revelo, A. Salazaret al. et al., "Levosimendan as a treatment for acute renal failure associated with cardiogenic shock after hip fracture," Brazilian Journal of Anesthesiology (English Edition), vol. 67, no. 1, pp. 89-91, 2017.

[15] M. Gooshe, M. Tabaeizadeh, A. R. Aleyasin et al., "Levosimendan exerts anticonvulsant properties against PTZ-induced seizures in mice through activation of nNOS/NO pathway: role for $\mathrm{K}_{\mathrm{ATP}}$ channel," Life Sciences, vol. 168, pp. 38-46, 2017.

[16] M. Okada and H. Yamawaki, "Levosimendan inhibits interleukin- $1 \beta$-induced apoptosis through activation of Akt and inhibition of inducible nitric oxide synthase in rat cardiac fibroblasts," European Journal of Pharmacology, vol. 769, pp. 86-92, 2015.
[17] G. Li, Y. Song, G. Li et al., "Downregulation of microRNA-21 expression inhibits proliferation, and induces G1 arrest and apoptosis via the PTEN/AKT pathway in SKM-1 cells," Molecular Medicine Reports, vol. 18, 2018.

[18] X. Bi, G. Zhang, X. Wang et al., "Endoplasmic reticulum chaperone GRP78 protects heart from ischemia/reperfusion injury through Akt activation," Circulation Research, vol. 122, no. 11, pp. 1545-1554, 2018.

[19] Y. Jiang, H. Chang, and G. Chen, "Effects of microRNA-20a on the proliferation, migration and apoptosis of multiple myeloma via the PTEN/PI3K/AKT signaling pathway," Oncology Letters, vol. 15, 2018.

[20] L. Zhao, X. Zhang, and S. Cui, "Matrine inhibits TPC-1 human thyroid cancer cells via the miR-21/PTEN/Akt pathway," Oncology Letters, vol. 16, 2018.

[21] M. Zhuang, X. Qiu, D. Cheng, C. Zhu, and L. Chen, "MicroRNA-524 promotes cell proliferation by down-regulating PTEN expression in osteosarcoma," Cancer Cell International, vol. 18, no. 1, 2018.

[22] Y.-P. Yuan, Z.-G. Ma, X. Zhang et al., "CTRP3 protected against doxorubicin-induced cardiac dysfunction, inflammation and cell death via activation of Sirt1," Journal of Molecular and Cellular Cardiology, vol. 114, pp. 38-47, 2018.

[23] F. Imam, et al.N. O. al-Harbi, M. M. al-Harbi et al., "Apremilast prevent doxorubicin-induced apoptosis and inflammation in heart through inhibition of oxidative stress mediated activation of NF- $\kappa \mathrm{B}$ signaling pathways," Pharmacological Reports, vol. 70, no. 5, pp. 993-1000, 2018.

[24] J. Gu, W. Hu, and D.-d. Zhang, "Resveratrol, a polyphenol phytoalexin, protects against doxorubicin-induced cardiotoxicity," Journal of Cellular and Molecular Medicine, vol. 19, no. 10, pp. 2324-2328, 2015.

[25] Z. Maayah, T. Zhang, M. Forrest et al., "DOX-Vit D, a novel doxorubicin delivery approach, inhibits human osteosarcoma cell proliferation by inducing apoptosis while inhibiting Akt and mTOR signaling pathways," Pharmaceutics, vol. 10, no. 3 , p. $144,2018$.

[26] T. Tian, X. Fu, J. Lu et al., "MicroRNA-760 inhibits doxorubicin resistance in hepatocellular carcinoma through regulating Notch1/Hes1-PTEN/Akt signaling pathway," Journal of Biochemical and Molecular Toxicology, vol. 32, no. 8, article e22167, 2018.

[27] J. Wang, H. Chen, Y. Zhou, Q. Su, T. Liu, and L. Li, "Levosimendan pretreatment inhibits myocardial apoptosis in swine after coronary microembolization," Cellular Physiology and Biochemistry, vol. 41, no. 1, pp. 67-78, 2017.

[28] C. Zhang, Z. Guo, H. Liu, Y. Shi, and S. Ge, "Influence of Levosimendan Postconditioning on Apoptosis of Rat Lung Cells in a Model of Ischemia-Reperfusion Injury," PLOS ONE, vol. 10, no. 1, p. e0114963, 2015.

[29] Z. W. Long, J. H. Wu, C. Hong, Y. N. Wang, and Y. Zhou, "MiR-374b promotes proliferation and inhibits apoptosis of human GIST cells by inhibiting PTEN through activation of the PI3K/Akt pathway," Molecules and Cells, vol. 41, no. 6, pp. 532-544, 2018.

[30] X.-F. Yin, Q. Zhang, Z.-Y. Chen et al., "NLRP3 in human glioma is correlated with increased WHO grade, and regulates cellular proliferation, apoptosis and metastasis via epithelial-mesenchymal transition and the PTEN/AKT signaling pathway," International Journal of Oncology, vol. 53, 2018. 
[31] W. Xu, Z. Yang, C. Xie et al., "PTEN lipid phosphatase inactivation links the hippo and PI3K/Akt pathways to induce gastric tumorigenesis," Journal of Experimental \& Clinical Cancer Research, vol. 37, no. 1, p. 198, 2018.

[32] Y. Wang, X. Sun, K. Ji et al., "Sirt3-mediated mitochondrial fission regulates the colorectal cancer stress response by modulating the Akt/PTEN signalling pathway," Biomedicine \& Pharmacotherapy, vol. 105, pp. 1172-1182, 2018.

[33] Z. Li, Z. Cheng, Y. Haifeng, M. Chen, and L. Li, "PTEN signaling inhibitor VO-OHpic improves cardiac myocyte survival by mediating apoptosis resistance in vitro," Biomedicine \& Pharmacotherapy, vol. 103, pp. 1217-1222, 2018.

[34] G. Gao, Y. Bian, H. Qian et al., "Gambogic acid regulates the migration and invasion of colorectal cancer via microRNA21-mediated activation of phosphatase and tensin homolog," Experimental and Therapeutic Medicine, 2018.

[35] J. Liu, W. Chen, H. Zhang, T. Liu, and L. Zhao, "miR-214 targets the PTEN-mediated PI3K/Akt signaling pathway and regulates cell proliferation and apoptosis in ovarian cancer," Oncology Letters, 2017.

[36] Y. Xing, M.-M. Wang, Y.-S. Feng, F. Dong, and F. Zhang, "Possible involvement of PTEN signaling pathway in the anti-apoptotic effect of electroacupuncture following ischemic stroke in rats," Cellular and Molecular Neurobiology, vol. 38, no. 8, pp. 1453-1463, 2018.

[37] H. Chen, L. Gao, Z. Huang et al., "C1qTNF-related protein 1 attenuates doxorubicin-induced cardiac injury via activation of AKT," Life Sciences, vol. 207, pp. 492-498, 2018.

[38] W. Chang, Q.-Q. Wu, Y. Xiao et al., "Acacetin protects against cardiac remodeling after myocardial infarction by mediating MAPK and PI3K/Akt signal pathway," Journal of Pharmacological Sciences, vol. 135, no. 4, pp. 156-163, 2017.

[39] H. Merino and D. K. Singla, "Secreted frizzled-related protein2 inhibits doxorubicin-induced apoptosis mediated through the Akt-mTOR pathway in soleus muscle," Oxidative Medicine and Cellular Longevity, vol. 2018, 11 pages, 2018.

[40] S. Wang, Y. Zheng, Z. He, W. Zhou, Y. Cheng, and C. Zhang, "SH2B1 promotes NSCLC cell proliferation through PI3K/Akt/mTOR signaling cascade," Cancer Cell International, vol. 18, no. 1, 2018.

[41] X. Zhang, Y. Yang, and Z. Feng, "Suppression of microRNA495 alleviates high-glucose-induced retinal ganglion cell apoptosis by regulating Notch/PTEN/Akt signaling," Biomedicine \& Pharmacotherapy, vol. 106, pp. 923-929, 2018.

[42] E. Grossini, K. Bellofatto, S. Farruggio et al., "Levosimendan inhibits peroxidation in hepatocytes by modulating apoptosis/autophagy interplay," PLOS ONE, vol. 10, no. 4, article e0124742, 2015.

[43] M. A. Ibrahim, S. A. Abdel-Gaber, E. F. Amin, S. A. Ibrahim, R. K. Mohammed, and A. M. Abdelrahman, "Molecular mechanisms contributing to the protective effect of levosimendan in liver ischemia-reperfusion injury," European Journal of Pharmacology, vol. 741, pp. 64-73, 2014.

[44] W.-J. M. Schellekens, H. van Hees, M. Linkels et al., "Levosimendan affects oxidative and inflammatory pathways in the diaphragm of ventilated endotoxemic mice," Critical Care, vol. 19, no. 1, p. 69, 2015. 